\title{
Preparation and Evaluation of Chrysin Encapsulated in PLGA- PEG Nanoparticles in the T47-D Breast Cancer Cell Line
}

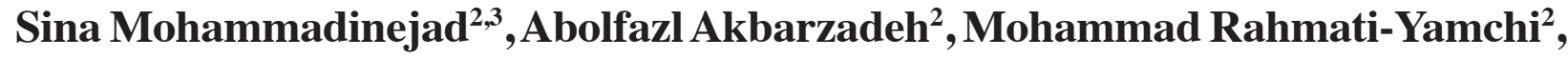 \\ Saeid Hatam ${ }^{5}$, Saeed Kachalaki ${ }^{4}$, Sanaat Zohreh' ${ }^{1}$, Nosratollah Zarghami1 ${ }^{1,2 *}$
}

\begin{abstract}
Background: Polymeric nanoparticles are attractive materials that have been widely used in medicine for drug delivery, with therapeutic applications. In our study, polymeric nanoparticles and the anticancer drug, chrysin, were encapsulated into poly (D, L-lactic-co-glycolic acid) poly (ethylene glycol) (PLGA-PEG) nanoparticles for local treatment. Materials and Methods: PLGA: PEG triblock copolymers were synthesized by ring-opening polymerization of $\mathrm{D}$, L-lactide and glycolide as an initiator. The bulk properties of these copolymers were characterized using $1 \mathrm{H}$ nuclear magnetic resonance spectroscopy and Fourier transform infrared spectroscopy. In addition, the resulting particles were characterized by scanning electron microscopy. Results: The chrysin encapsulation efficiency achieved for polymeric nanoparticles was $70 \%$ control of release kinetics. The cytotoxicity of different concentration of pure chrysin and chrysin loaded in PLGA-PEG (5-640 $\mu \mathrm{M})$ on T47-D breast cancer cell line was analyzed by MTT-assay. Conclusions: There is potential for use of these nanoparticles for biomedical applications. Future work should include in vivo investigation of the targeting capability and effectiveness of these nanoparticles in the treatment of breast cancer.
\end{abstract}

Keywords: Triblock copolymer - chrysin - encapsulation - drug encapsulation efficiency

Asian Pac J Cancer Prev, 16 (9), 3753-3758

\section{Introduction}

Cancer is becoming one of the most public health problems in the world; in 2008, Ferlay et al. estimated the occurrence of new cancer cases about 12.7 million and cancer related death nearly 7.6 million. The most commonlydiagnosed cancers in wild world are lung cancer (12.7\% of the total), breast cancer $(10.9 \%)$ and colorectal cancer $(9.7 \%)$, while the most common cancer related to death are lung cancer $(18.2 \%$ of the total), stomach cancer $(9.7 \%)$ and liver cancer $(9.2 \%)$ (Ferlay et al., 2010). inUnited States, occurrence of new breast cancer case alone is $29 \%$ (Siegel et al., 2012).

Common cancer therapies including surgery, chemotherapy and radiation are often insufficient in treating of cancer diseases, thoughdeveloping new methods of treatment or cancer therapy are necessary. Some plant medicines have been widely consumed for treatment of various diseases including cancer (Butler, 2004; Koehn and Carter, 2005). Polyphenolsaccount for an important group of plant constituents which contain up to 8,000 different well-known structures (Bravo, 1998).These phenolic components produced by plants are used as secondary metabolites. These components, upon their structure are classified into 10 different types: flavonoids, simple phenols, coumarins, phenolic acids, naphthoquinones, anthaquinones, lignins, isocoumarins, stilbenes and xanthones (Cook and Samman, 1996). The most important class of these phenolic constituents is flavonoids, whichis generally safe and shows low toxicity (Samarghandian et al., 2011; Khacha-Ananda et al., 2013). Chrysin (5,7-dihydroxyflavone)is a biologically active flavonoid extracted from plants, propolis and honey (Suganya et al., 2014).Chrysin possesses antiinflammatory, anti-oxidant and anti-allergic properties (Kadir et al., 2013). Many studies in recent yearsrevealed that chrysin is candidate for cancer therapy in various cancer cell lines by inhibition of cell proliferation and induction of apoptosis (Monasterio et al., 2004; Khoo et al., 2010; Sak, 2014). Chrysin treatment lead to a strong

${ }^{1}$ Hematology and Oncology Research Center, Tabriz University of Medical Sciences, Tabriz, Iran, ${ }^{2}$ Department of Medical Biotechnology, Faculty of Advanced Medical Sciences, ${ }^{3}$ International Aras Branch, ${ }^{4}$ Department of Immunology, Tabriz University of Medical Sciences, Tabriz, Iran, ${ }^{5}$ Department of Biomedical Research Center of Sheffield Hallam University (BMRC), Sheffield, United Kingdom*For correspondence: Zarghami@tbzmed.ac.ir. 
increase in p21 and accumulation of cells in G1 phase (Pal-Bhadra et al., 2012). Activate P21 is a cdk (Cyclindependent kinases) inhibitor, which lead to apoptosis or cell cycle arrest by reducing in cyclin and $\mathrm{cdk}(\mathrm{Cai}$ and Dynlacht, 1998).

Cell proliferation is strongly regulated by enormous interactions between molecules (Zhou et al., 2011). The regulatory protein called cyclin when joined toCDKs, promote the cell through the cell cycle (Dickson and Schwartz, 2009; Zeybek et al., 2013). Cyclin D1/ CDK2 and CDK4 transit cell from $\mathrm{G} 1$ to $\mathrm{S}$ phase, while cyclin E/ CDK2 and cyclin A/ CDK2 respectively, controls entry to $\mathrm{S}$ phase and controls $\mathrm{S}$ phase progression (Sherr and Roberts, 1999; Zhang et al., 2012).Overexpression of cyclin D1 has been reported to be directly associated with tumorigenicity, poor prognosis and resistance to therapy in several cancer cell lines including breast cancer (Hosokawa and Arnold, 1998; Yu et al., 2001; Fu et al., 2004). Several studies show that cyclin D1 is overexpressed in breast cancer up to $50 \%$ (Gillett et al., 1994; Ishii et al., 2006; Sui et al., 2014). Thus, inhibition of cyclin D1 provides an approach to treatment of breast cancer.

Although chrysin possesses anti-cancer properties but poor bioavailability of this phenolic compound is an important obstacle for cancer treatment (CAO et al., 2014), which can be due to low absorption, quick metabolism and rapid systemic elimination. One ofThe approaches to overcome this issue is use of nanoparticles (Yin et al., 2013). Biodegradable polymeric nanocapsules are massively in use in recent years to maintain the quality of many drugs and biologically active compounds. Nanoparticle encapsulation helps to protect therapeutic molecules from being prematurely degraded, enhances their solubility, and provides controlled drug targeting condition (Khalil et al., 2013). PLGA (poly lactic-coglycolic acid) is polymeric nanoparticle that shows high degree of biocompatibility and Biodegradability as well as safety for human, which is approved by U.S. Food and Drug Administration (FDA) (Alimohammadi and Joo, 2014). Surface modification of PLGA with PEG (polyethylene glycol) could improve permeability and half-life of circulation (Dwivedi et al., 2014). Chrysin to be loaded in PLGA_PEG nanoparticles could increase its bioavailability and efficiency of anti-cancer properties (Braden et al., 2008).

In this study we hypothesize that encapsulation of chrysin with PLGA-PEG have strong effect in cell cycle arrestcompared to the effect of pure chrysin and we investigate the efficacy of chrysin loaded in PLGA-PEG in inhibition of cell proliferation and reduction of cyclinD1.

\section{Materials and Methods}

\section{Materials}

Chrysin, penicillin G, streptomycin, glycolide, PEG (6000), 3(4, 5-dimethylthiazol-2-yl) 2, 5-diphenyltetrazolium bromide (MTT), stannous octoate (Sn (Oct) 2), dimethyl sulphoxide (DMSO), dichloromethane (DCM), polyvinyl alcohol (PVA) and D, L-lactide were purchased from Sigma-Aldrich (USA). T47-D breast cancer cell line (code: c203) was obtained from Pasteur Institute of Iran. Trypsin-EDTA, Fetal bovine serum (FBS) and RPMI1640 were from Gibco, Invitrogen (UK).Primers were purchased from Takapouzist. 2-step RT-PCR kit obtained from vivantis while, Hot TaqEvaGreenqPCR Mix and RNX-Plus kit purchased from fromCinnaGen (Iran) and used for cDNA synthesis, real time PCR and total RNA extraction respectively. Real-time PCR was done using Corbett (Rotor Gene 6000). Nanodrop spectrophotometer was Bio Photometer. KYKY model EM3200 and Fourier transform infrared spectroscopy (FTIR) Perkin Elmer Series was used for Scanning electron microscopy (SEM) and Infrared spectra respectively.

\section{Synthesis of PLGA-PEG}

Synthesis of PLGA-PEG was done through ring open polymerization of glycolide and DL lactide followed by addition of PEG6000. As stated in melt polymerization procedure under vaccume, PEG 6000 and PLGA were copolymerized in presence of stannous octoate [Sn (Oct) 2] as the catalyst. DL-lactide (2.882g), PEG6000 $1.54 \mathrm{~g} \mathrm{(45 \%}$ $\mathrm{w} / \mathrm{w})$ and glycolide $(0.570 \mathrm{~g})$ were melted in bottleneck flask in 140 Celsius degrees under a nitrogen atmosphere. Reaction mixture comprising a 3; 1 proportion of DLlactide to glycolide and $0.05 \%(\mathrm{w} / \mathrm{w})$ stannous octoate was prepared and heated to $180^{\circ} \mathrm{C}$ and maintained for four hours.

\section{Chrysin encapsulation with PLGA-PEG}

Chrysin were loaded in PLGA-PEG nanoparticle by using s/o/w technique. Briefly, PLGA-PEG (200 mg) was dissolved in dichloromethane (DCM) while pure chrysin (20 mg) was added to this solution and sonicated for 1 minute to yield the s/o primary emulsion. Dimethyl sulphoxide (DMSO) and polyvinyl alcohol (PVA) $1 \%$ $(1: 1)$ was added to $\mathrm{s} / \mathrm{o}$ emulsion then sonicated for 1 minute to produces/o/w emulsion. Subsequentlysolvents of this emulsion were evaporated with rotary evaporator then this emulsion was centrifuged 30 minutes at $10000 \times \mathrm{g}$. The drug encapsulation efficiency of chrysin loaded in PLGA-PEG was measured by assessing the supernatant of centrifuged emulsion at $348 \mathrm{~nm}$ with spectrophotometer (Braden et al., 2008). Encapsulation efficiencymeasured by this formula:

$$
\begin{aligned}
\text { Drug }_{\text {encapsulated }} & =\text { Drug }_{\text {total }}-\text { Drug }_{\text {filtrate }} \\
\text { Encapsulation } \% & =\left(\frac{\text { Drug }_{\text {encapsulated }}}{\text { Drug }_{\text {total }}}\right) \times 100
\end{aligned}
$$

\section{FTIR analysis}

Functional groups were characterized using FTIR analysis. Figure 1 shows that absorption band at 3509.9 $\mathrm{cm}^{-1}$ is assigned to terminal hydroxyl groups in the copolymer from which PEG homopolymer has been removed. The bands at $3010 \mathrm{~cm}^{-1}$ and $2955 \mathrm{~cm}^{-1}$ are due to $\mathrm{C}-\mathrm{H}$ stretch of $\mathrm{CH}$, and $2885 \mathrm{~cm}^{-1}$ due to $\mathrm{C}-\mathrm{H}$ stretch of $\mathrm{CH}$. A strong band at $1762.6 \mathrm{~cm}^{-1}$ is assigned to $\mathrm{C}=\mathrm{O}$ stretch. Absorption at $1186-1089.6 \mathrm{~cm}^{-1}$ is due to $\mathrm{C}-\mathrm{O}$ stretch. 


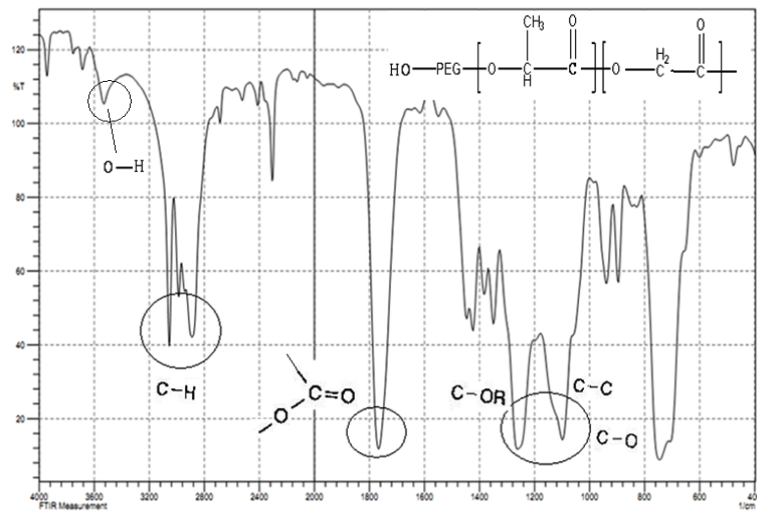

Figure 1. FTIR Result show Presence of Chrysin PLGA-PEG Nanoparticle
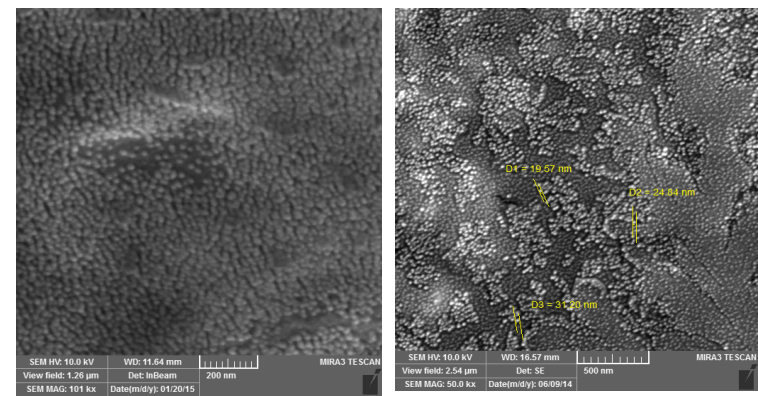

Figure 2. SEM picture show (a) the size PLGA-PEG and (b)drug loaded in PLGA-PEG nanoparticle

\section{SEM analysis}

Measurement of size and checking the morphology of nanoparticles were performed by scanning electron microscopy (SEM). The nanographs of PLGA-PEG polymeric nanoparticles (Figure 2A), and Chrysin -loaded PLGA-PEG copolymers nanoparticles are shown. Observing the photograph, it can be seen that the nanoparticles were well aggregated, which was due to the Nano-size of the polymeric nanoparticles about $15 \mathrm{~nm}$. After encapsulation, the size of Chrysin -loaded PLGA-PEG copolymers nanoparticles with PLGA-PEG copolymers, changed to $20-75 \mathrm{~nm}$ and dispersion of the particles was greatly improved (Figure 2B),which can be explained by the electrostatic repulsion force and steric hindrance between the copolymer chains on the encapsulated polymeric nanoparticles. The samples were coated with gold particles.

\section{Cell culture}

T47-D breast cancer cell line were cultured in RPMI1640 medium with $10 \% \mathrm{FBS}$ and incubated at $37^{\circ} \mathrm{C}$ and $5 \% \mathrm{CO}_{2}$.

The RPMI-1640 also has streptomycin $(50 \mu \mathrm{g} / \mathrm{mL})$, penicillin $\mathrm{G}(100 \mathrm{U} / \mathrm{mL})$ and $\mathrm{NaHCO}_{3}(2 \mathrm{mg} / \mathrm{mL})$.

\section{Cell viability analysis}

MTT assay was done to measure T47-D cell viability. 10000 cell/well were seeded on 96-well plate and allowed them to attach for $24 \mathrm{~h}$. Afterward cells were treated with different concentration of pure and Nano chrysin (5-640 $\mu \mathrm{M})$ for 24,48 and $72 \mathrm{~h}$. For each concentration triplicate
Table 1. The Raction Mixtures Incubated for RealTime PCR in Following Order

\begin{tabular}{|c|c|c|c|}
\hline step & Temperature & \multicolumn{2}{|c|}{ Number of cycle } \\
\hline \multicolumn{4}{|c|}{ Initial denaturation } \\
\hline & $95^{\circ} \mathrm{C}$ & 15 minutes & 1 cycle \\
\hline \multicolumn{4}{|c|}{ Denaturation } \\
\hline & $95^{\circ} \mathrm{C}$ & 15 seconds & 45 cycles \\
\hline \multicolumn{4}{|c|}{ Annealing } \\
\hline & $59^{\circ} \mathrm{C}$ & 30 seconds & \\
\hline \multicolumn{4}{|c|}{ Elongation } \\
\hline & $72^{\circ} \mathrm{C}$ & 20 seconds & \\
\hline \multicolumn{4}{|c|}{ Melting } \\
\hline & $59-95^{\circ} \mathrm{C}$ & 5 seconds for each degrees & 1 cycle \\
\hline
\end{tabular}

were chosen and control received same amount of solvent (DMSO). After 24, 48 and $72 \mathrm{~h}$ medium were removed from all well while, $200 \mu \mathrm{L}$ medium and $50 \mu \mathrm{L}$ MTT solution were added to each wells and incubated for 4 $\mathrm{h}$ at $37^{\circ} \mathrm{C}$. Then mixture of MTT solution and medium discarded from all wells and MTT crystals were dissolved by adding $200 \mu \mathrm{L}$ DMSO and $50 \mu \mathrm{L}$ Sorenson's buffer. The viability of the cells was calculated by this formula:

$$
\text { cell viability } \%=\left(\frac{\text { absorbance of expremental wells }}{\text { absorbance of control wells }}\right) \times 100
$$

\section{RNA extraction, cDNAsynthesis and real-time PCR}

T47-D cells were treated with different concentrations of pure and Nano chrysin $(34,54$ and $74 \mu \mathrm{M})$ for $24 \mathrm{~h}$. After drug exposure time, according to the instructions of the RNX-Plus manufacturer total RNA were extracted. Nanodrop was proved the purity and quantity of total RNA.After the integrity of total RNA was examined by using electrophoresis, complementary DNA (cDNA) was synthesis by 2-step RT-PCR kit according to the instructions of the manufacturer. Next Quantitative real-time PCR technique was used to determine cyclin D1 expression levels and Hot TaqEvaGreenqPCR Mix used following the instructions of the manufacturer. Sequence of forward and reverse primers for cyclin D1 were, F: 5'-TGCCCTCTGTGCCACAGATG-3', R: 5'-TCTGGAGAGGAAGCGTGTGA-3' and primers for $\beta$-actin, F: 5'-TCCCTGGAGAAGAGCTACG-3', R: 5'-GTAGTTTCGTGGATGCCACA-3'. The samples were incubated in following order (Table 1). Relative cyclin D1 expression levels was normalized by housekeeping gene $(\beta$-actin) and relative expression of cyclin D1 calculated by this formula:

$$
\text { Relative gene expression }=\frac{\mathrm{E}(\text { target })^{\Delta \mathrm{CP}(\text { target })}}{\mathrm{E}(\text { reference })^{\Delta \mathrm{CP}(\text { refrence })}}
$$

$\mathrm{E}_{\text {target }}$ is referred to real-time efficiency of target gene transcript while $\mathrm{E}_{\text {reference }}$ is referred to real-time efficiency of reference gene transcript. $\triangle \mathrm{CP}$ target isreferredto $\mathrm{CP}$ deviation of (control - sample) of the target gene transcript and $\triangle \mathrm{CP}$ reference is referred to $\mathrm{CP}$ deviation of (control sample) of the reference gene transcript. 
Table 2. IC $_{50}$ Value of Pure and Nano Chrysin

\begin{tabular}{llll}
\hline Different concentrations of chrysin $(\mu \mathrm{M})$ & $24 \mathrm{~h}$ & $48 \mathrm{~h}$ & $72 \mathrm{~h}$ \\
Free chrysin & 54.56 & 46.74 & 40.28 \\
Nano chrysin & 48.72 & 44.78 & 37.54 \\
\hline
\end{tabular}

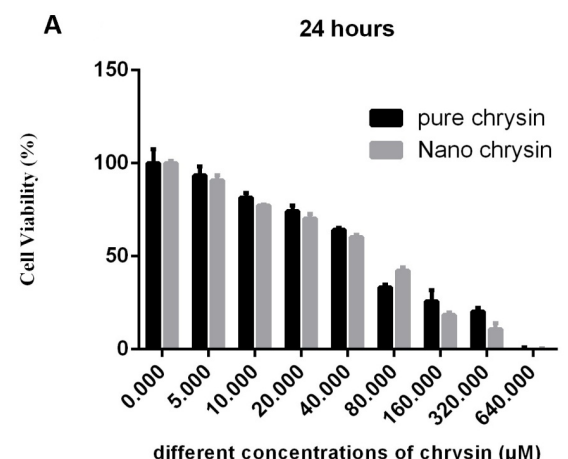

B

48 hours

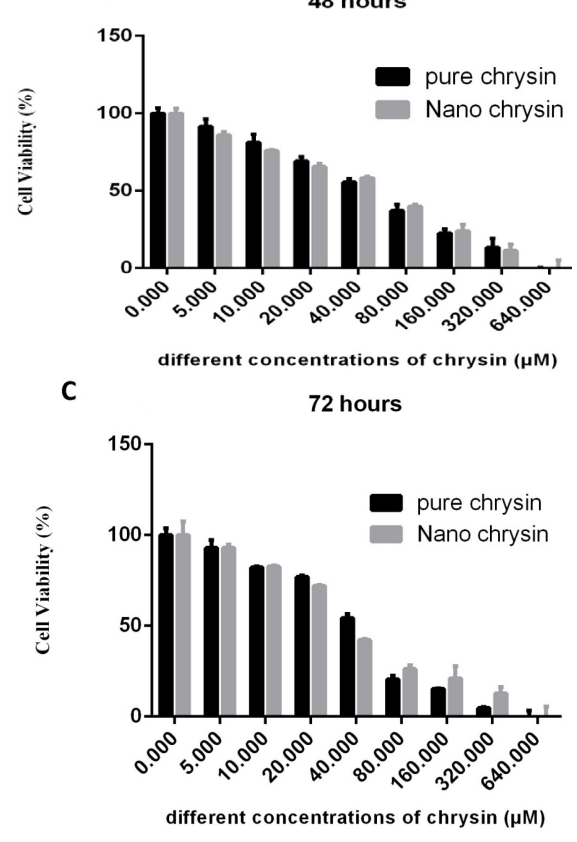

Figure 3. Compared Different Concentration of Pure and Nano Chrysin for 24, 48 and 72 Hours. A: Related to 24 Hours Treatment, B and C Also Related to Chrysin Treatment for $\mathbf{4 8}$ and $\mathbf{7 2}$ hours, Respectively

\section{Data analysis and statistics}

GraphPad.Prism.6.01. was employed for all data analysis and plotting graphs. Statistical analysis was performed by ANOVA test (by one-way analysis of variance). The result was assumed of statistical importance when/if pvalue was smaller than 0.05 .

\section{Results}

\section{Encapsulation efficiency}

Analysis of supernatant of chrysin loaded in PLGAPEG showed that chrysin were encapsulated via PLGA-PEG nanoparticle in $98.6 \%$ encapsulation efficiency. Success in PLGA-PEG synthesis by open ring copolymerization was confirmed by SEM (Figure2). The results of FTIR verified present of chrysin in PLGA-PEG.

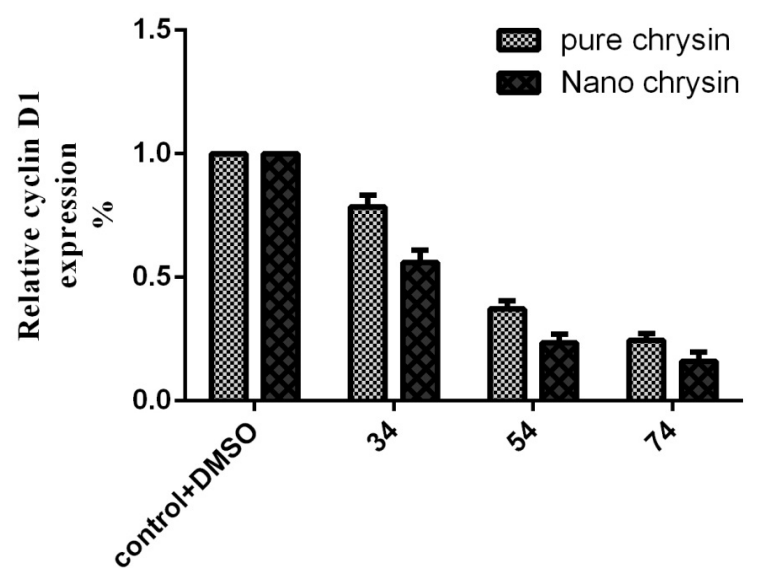

Different concentrations of chrysin $(\mu \mathrm{M})$

Figure 4. Real-Time PCR Results Showed that Relative Expression of Cyclin D1 were Decreased by Pure and Nano Chrysin

The results of FTIR verified present of chrysin in PLGAPEG (Figure 1). Morphological feature of PLGA-PEG was analysis by SEM (Figure 2).

\section{Cell viability assay}

The cytotoxicity of different concentration of pure chrysin and chrysin loaded in PLGA-PEG $(5-640 \mu \mathrm{M})$ on T47-D breast cancer cell line were analyzed by MTTassay technique. These serial dilution of pure and Nano chrysin were treated on T47-D for 24, 48 and $72 \mathrm{~h}$ and results suggested that pure chrysin and chrysin loaded in PLGA-PEG could inhibit cell proliferation by dose dependent manner. The $\mathrm{IC}_{50}$ value for $24 \mathrm{~h}$ treatments were 54.56 for pure chrysin and 48.72 for chrysin loaded in PLGA-PEG (Figure $3 \mathrm{~A}$ ). $\mathrm{IC}_{50}$ value of $48 \mathrm{~h}$ were 46.74 and 44.78 respectively for pure and Nano chrysin (Figure $3 \mathrm{~B}$ ) while for $72 \mathrm{~h}$ treatment of pure chrysin and Nano chrysin $\mathrm{IC}_{50}$ value were 40.28 and 37.54 respectively (Figure 3C). These results showed that PLGA-PEG nanoparticle could improve the efficiency of chrysin in cell growth inhibition (Table 2).

\section{Quantitative real-time PCR}

The levels of cyclin D1 expression was measured by real-time PCR. Cyclin D1 mRNA levels were normalized by $\beta$-actin (housekeeping gene). Different concentration of pure chrysin $(34,54$ and $74 \mu \mathrm{M})$ for 24 treatment decreased relative cyclin D1 expression in .78 $\pm .04, .37 \pm .03$ and $24 \pm .02$ respectively. Whereas chrysin loaded in PLGAPEG nanoparticle in same concentration reduced further relative mRNA expression of cyclin D1 in .56 $\pm .04,23 \pm .03$ and $.16 \pm .03$ for 34,54 and $74 \mu \mathrm{M}$ respectively (Figure 4 ). The results suggested that chrysin loaded in PLGA-PEG had better effect in decline cyclin D1 expression rather than pure chrysin.

\section{Discussion}

Breast cancer is one of the most common types of cancer with $>1,300,000$ cases and 450,000 mortalities 
annually worldwide (27-1). Although fatalities due to breast cancer are decreasing, breast cancer remains the second leading cause of cancer related mortality among females (27-2, 3).Surgical removal of the primary tumor, chemotherapy and radiotherapy are inadequate for cancer treatment. This high $\neg$ lights the requirement for therapies that are able to treat the advanced stages of the disease. Improvement in the understanding of the molecular mechanisms involved in cancer has led to the identification of novel targets and the development of specific therapies, which are referred to as targeted therapies. The cell cycle is a promising target involved in cancer growth. Cyclin D1 is proto-oncogenes that over express in many cancers, in which drive the cells from G1 to $\mathrm{S}$ phase also play important role in tumorigenicity and cancer progression $(6,7)$. Medicinal plants have been used worldwide and have been demonstrated to be a source of effective anticancer agents (8). Chrysin is phenolic compound that extract form honey and shows anti-cancer properties and down regulated cyclin D1 expression in many cancer cell line. Due to poor oral bioavailability, chrysin may not be successfully used as a dietary flavonoid for cancer chemo $\neg$ therapeutics (19-9). One of the most promising applications of current nanotechnology is in targeted drug delivery to treat cancer. PLGA-PEG is favorable nanoparticle for drug delivery and safety of this nanoparticle approved by FDA (44).

Breast cancer takes about half a million victims a year in the world and is one of the most incident types (Network, 2012). However, mortalities associated with this cancer are being decreased but still this annually is the second leading cancer-associated mortality cause among women of the world (DeSantis et al., 2011). Routine methods such as surgical removal of the tumor and chemotherapy seem inadequate for treating breast cancer. This again reminds the requirement for treatment strategies which make treatment proficient in advanced stages of cancer. Enhancements in the knowledge about the molecular mechanisms concerned with cancer has resulted in the identification of novel targets and development of more specific therapies, also referred to as targeted therapies (de Bono et al., 2003). The cell cycle is a promising target involved in cancer growth. Cyclin D1 is proto-oncogenes that over express in many cancers, in which drive the cells from $\mathrm{G} 1$ to $\mathrm{S}$ phase also play important roles in tumorigenicity and cancer progression (Schwartz and Shah, 2005; Malumbres and Barbacid, 2009).

Plants with medical values have been investigated worldwide and demonstrated to be a source of effective anticancer metabolites (Chin et al., 2006). Chrysin is phenolic compound that is extract form honey showing anti-cancer properties and is accepted to have downregulating effects on cyclin D1 expression in many cancer cell lines. Due to poor oral bioavailability, chrysin may not be successfully used as a dietary flavonoid for cancer chemotherapeutics (Walle et al., 2001). One of the most promising applications of current nanotechnology is in targeted drug delivery to treat cancer. PLGA-PEG is favorable nanoparticle for drug delivery and safety of this nanoparticle approved by FDA (Khalil et al., 2013).

In this study we suggested that, chrysin encapsulated through PLGA-PEGhad greater bioavailability and effectiveness of chrysin in cell growth inhibition was enhanced. Boon Yin Khoo et al. used phosphorylated chrysin to develop the effectiveness of anti-cancer properties, although chrysin could inhibit proliferation and induced apoptosis but diethyl chrysin-7-yl phosphate (CPE) and tetraethyl bis-phosphoric ester of chrysin (CP) have more potent have more affect in cell progression(Zhang et al., 2004). Other studies shown that, the cytotoxicity of chrysin more than other analogs and these properties come from two hydroxyl groupin AC rings of flavone were responsible for effective cytotoxicity and modification of -OH groups in flavone could harm potential of cytotoxicity (Chang et al., 2008). While we used chrysin loaded in PLGA-PEG nanoparticle to maintenance the anti-cancer effects of this dietary flavonoids. In parallel to our works Manika Pal-Bhadra and colleague shown that chrysin arrest the cell cycle and reduce cyclin D1 expression in skin cancer. Whereas we showed that chrysin could decreased cyclin D1 levels but Nano chrysin $(74 \mu \mathrm{M})$ down regulated cyclin D1 mRNA up to $80 \%$ (Pal-Bhadra et al., 2012).

In conclusion: chrysin encapsulated with PLGA-PEG show significant repression effect in cell growth rather than chrysin alone and PLGA-PEG is promising approach for breast cancer therapy. Obviously, our results are limited to a number of cell lines in vitro which should be further accredited by testing the drug on live animal models(with enough replicates).

\section{Acknowledgements}

Department of Medical Biotechnology, Faculty of Advanced Medical Sciences, Tabriz University of Medical Sciences, for all support provided. This work is funded by 92/20 grant of Hematology and Oncology Research Center, Tabriz University of Medical Sciences, Tabriz, Iran.

\section{References}

Alimohammadi YH, Joo SW (2014). PLGA-based nanoparticles as cancer drug delivery systems. Asian Pac J Cancer Prev, 15, 517-35.

Bravo L (1998). Polyphenols: chemistry, dietary sources, metabolism, and nutritional significance. Nut Rev, 56, 317-33.

Butler MS (2004). The role of natural product chemistry in drug discovery. J Nat Prod, 67, 2141-53.

Cai K, Dynlacht BD (1998). Activity and nature of p21WAF1 complexes during the cell cycle. Proc Nat Acad Sci USA, 95, 12254-9.

Cao XZ, Xiang HL, Quan MF, et al (2014). Inhibition of cell growth by BrMC through inactivation of Akt in HER-2/neuoverexpressing breast cancer cells. Oncol Letters, 7, 1632.

Chang H, Mi M, Ling W, et al (2008). Structurally related cytotoxic effects of flavonoids on human cancer cells in vitro. Arch Pharm Res, 31, 1137-44.

Chin YW, Balunas MJ, Chai HB, et al (2006). Drug discovery from natural sources. AAPS J, 8, 239-53.

Cook N, Samman S (1996). Flavonoids-chemistry, metabolism, cardioprotective effects, and dietary sources. $J$ Nutr $B, 7$, 66-76. 


\section{Mohammadinejad Sina et al}

de Bono JS, Tolcher AW, Rowinsky EK (2003). The future of cytotoxic therapy: selective cytotoxicity based on biology is the key. Breast Cancer Res, 5, 154-9.

DeSantis C, Siegel R, Bandi P, et al (2011). Breast cancer statistics, 2011. CA: Cancer J Clin, 61, 408-18.

Dickson M, Schwartz G (2009). Development of cell-cycle inhibitors for cancer therapy. Current Oncol, 16, 36.

Dwivedi M, Sharma S, Shukla P, et al (2014). Development and evaluation of anticancer polymeric nano-formulations containing curcumin and natural bioenhancers. J Biomaterials Tissue Eng, 4, 198-202.

Ferlay J, Shin HR, Bray F, et al (2010). Estimates of worldwide burden of cancer in 2008: GLOBOCAN 2008. Int J Cancer, 127, 2893-917.

Fu M, Wang C, Li Z, et al (2004). Minireview: Cyclin D1: normal and abnormal functions. Endocrinol, 145, 5439-47.

Gillett C, Fantl V, Smith R, et al (1994). Amplification and overexpression of cyclin D1 in breast cancer detected by immunohistochemical staining. Cancer Res, 54, 1812-7.

Ghasemal S, Nejati-Koshki K, Akbarzadeh A, et al (2013). Inhibitory effects of $B$-cyclodextrin-helenalin complexes on H-TERT gene expression in the T47D breast cancer cell line - Results of real time quantitative. Asian Pac J Cancer Prev, 14, 6949-53.

Ishii Y, Pirkmaier A, Alvarez JV, et al (2006). Cyclin D1 overexpression and response to bortezomib treatment in a breast cancer model. J Nati Cancer Inst, 98, 1238-47.

Kadir EA, Sulaiman SA, Yahya NK, et al (2013). Inhibitory effects of Tualang honey on experimental breast cancer in rats: a preliminary study. Asian Pac J Cancer Prev, 14, 2249-54.

Khacha-Ananda S, Tragoolpua K, Chantawannakul P, et al (2013). Antioxidant and Anti-cancer Cell Proliferation Activity of Propolis Extracts from Two Extraction Methods. Asian Pac J Cancer Prev, 14, 6991-5.

Khalil NM, Nascimento TCFd, Casa DM, et al (2013). Pharmacokinetics of curcumin-loaded PLGA and PLGAPEG blend nanoparticles after oral administration in rats. Colloids Surfaces B Biointerfaces, 101, 353-60.

Khoo BY, Chua SL, Balaram P (2010). Apoptotic effects of chrysin in human cancer cell lines. Int J Mol Sci, 11, 2188-99.

Koehn FE, Carter GT (2005). The evolving role of natural products in drug discovery. Nature Rev Drug Disc, 4, 206-20.

Malumbres M, Barbacid M (2009). Cell cycle, CDKs and cancer: a changing paradigm. Nat Rev Cancer, 9, 153-66.

Monasterio A, Urdaci MC, Pinchuk IV, et al (2004). Flavonoids induce apoptosis in human leukemia U937 cells through caspase-and caspase-calpain-dependent pathways. $\mathrm{Nut}$ Cancer, 50, 90-100.

Network CGA (2012). Comprehensive molecular portraits of human breast tumours. Nature, 490, 61-70.

Pal-Bhadra M, Ramaiah MJ, Reddy TL, et al (2012). Plant HDAC inhibitor chrysin arrest cell growth and induce p21WAF1 by altering chromatin of STAT response element in A375 cells. BMC Cancer, $\mathbf{1 2}, 180$.

Sak K (2014). Characteristic features of cytotoxic activity of flavonoids on human cervical cancer cells. Asian Pac J Cancer Prev, 15, 8007-19.

Samarghandian S, Afshari JT, Davoodi S (2011). Chrysin reduces proliferation and induces apoptosis in the human prostate cancer cell line pc-3. Clin, 66, 1073-9.

Schwartz GK, Shah MA (2005). Targeting the cell cycle: a new approach to cancer therapy. J Clin Oncol, 23, 9408-21.

Sherr CJ, Roberts JM (1999). CDK inhibitors: positive and negative regulators of $\mathrm{G} 1$-phase progression. Genes Development, 13, 1501-12.

Siegel R, Naishadham D, Jemal A (2012). Cancer statistics, 2012.
Ca: Cancer J Clin, 62, 10-29.

Suganya J, Radha M, Naorem DL, et al (2014). In silico docking studies of selected flavonoids-natural healing agents against breast cancer. Asian Pac J Cancer Prev, 15, 8155-9.

Sui JQ, Xie KP, Zou W, et al (2014). Emodin inhibits breast cancer cell proliferation through the ERalpha-MAPK/Aktcyclin D1/Bcl-2 signaling pathway. Asian Pac J Cancer Prev, 15, 6247-51.

Walle T, Otake Y, Brubaker J, et al (2001). Disposition and metabolism of the flavonoid chrysin in normal volunteers. British J Clin Pharm, 51, 143-6.

Yin H-T, Zhang D, Wu X, et al (2013). In vivo evaluation of curcumin-loaded nanoparticles in a A549 xenograft mice model. Asian Pac J Cancer Prev, 14, 409-12.

Yu Q, Geng Y, Sicinski P (2001). Specific protection against breast cancers by cyclin D1 ablation. Nat, 411, 1017-21.

Zeybek U, Yaylim I, Ozkan NE, et al (2013). Cyclin D1 gene G870A variants and primary brain tumors. APJCP, 14, 4101-6.

Zhang T, Chen X, Qu L, et al (2004). Chrysin and its phosphate ester inhibit cell proliferation and induce apoptosis in Hela cells. Bioorgan Med Chem, 12, 6097-105.

Zhang YY, Xu ZN, Wang JX, et al (2012). G1/S-specific cyclin-D1 might be a prognostic biomarker for patients with laryngeal squamous cell carcinoma. Asian Pac J Cancer Prev, 13, 2133-7.

Zhou QM, Wang XF, Liu XJ, et al (2011). Curcumin enhanced antiproliferative effect of mitomycin $\mathrm{C}$ in human breast cancer MCF-7 cells in vitro and in vivo. Acta Pharmacol Sinica, 32, 1402-10. 